\title{
On Projective Planes Of Order Nine
}

\author{
By Marshall Hall, Jr., J. Dean Swift and Raymond Killgrove
}

1. Introduction. There are four known projective planes of order nine: the desarguesian plane; the plane coordinatizable by a Veblen-Wedderburn system which is not a field; its dual; and a self-dual non-desarguesian plane. The last was originally found by Veblen and Wedderburn [4]; its structure has been generalized by Hughes [3].

Each of these planes was known to have an elementary abelian addition in an appropriate ternary ring. A natural question was whether or not there were any other planes with this property. A search was made partly by hand, partly by machine for such planes. The result of the search is that no further planes exist with this property but that the three non-desarguesian planes all may be coordinatized in more than one way with an elementary abelian addition. For notation and terminology used in this paper the reader is referred to [1].

2. The additive pencil and lines consistent with it. In a plane of order 9 with an elementary abelian addition we may assume initially three pencils of finite lines $x=c, y=c$, and $y=x+c, c=0,1, \cdots, 8$, where the addition in the last pencil is that of the elementary abelian group. Specifically this last pencil may be represented by the latin square:

$\begin{array}{lllllllll}0 & 1 & 2 & 3 & 4 & 5 & 6 & 7 & 8 \\ 1 & 2 & 0 & 4 & 5 & 3 & 7 & 8 & 6 \\ 2 & 0 & 1 & 5 & 3 & 4 & 8 & 6 & 7 \\ 3 & 4 & 5 & 6 & 7 & 8 & 0 & 1 & 2 \\ 4 & 5 & 3 & 7 & 8 & 6 & 1 & 2 & 0 \\ 5 & 3 & 4 & 8 & 6 & 7 & 2 & 0 & 1 \\ 6 & 7 & 8 & 0 & 1 & 2 & 3 & 4 & 5 \\ 7 & 8 & 6 & 1 & 2 & 0 & 4 & 5 & 3 \\ 8 & 6 & 7 & 2 & 0 & 1 & 5 & 3 & 4\end{array}$

where, as is customary, in each row of the square: $y_{0} y_{1} y_{2} y_{3} y_{4} y_{5} y_{6} y_{7} y_{8}$ is to be read as the second row of the permutation:

$$
\left(\begin{array}{ccccccccc}
0 & 1 & 2 & 3 & 4 & 5 & 6 & 7 & 8 \\
y_{0} & y_{1} & y_{2} & y_{3} & y_{4} & y_{5} & y_{6} & y_{7} & y_{8}
\end{array}\right)
$$

and represents a line whose nine finite points are $\left(i, y_{i}\right) i=0,1, \cdots, 8$.

In our search it is sufficient to find the affine planes of order nine; the extension to projective planes by adding the points at infinity is trivial.

It turns out that there are 2241 lines consistent with the pencil (2.1), these being represented by permutations $(2.2)$ which do not have as many as two points $\left(i, y_{i}\right)$ in common with any one of the lines of (2.1). In practice the lines for which $y_{0}=0$

Received April 1, 1959. The work of Professor Hall was supported in part by the Office of Ordnance Research; that of Professor Swift and Mr. Killgrove and the preparation of this paper were supported in part by the Office of Naval Research. 
are first found. These are then subjected to the permutations given by (2.1) to obtain the full list. If $L$ is a consistent line and $A_{a}$ is a permutation of (2.1) then $L A_{a}$ is also and conversely. Indeed if $L_{1}$ and $L_{2}$ are any two lines and $P$ any permutation, the number of intersections (coincidences) of $L_{1}$ and $L_{2}$ is obviously equal to the number for $L_{1} P$ and $L_{2} P$. In our case the group goes into itself under multiplication by $A_{a}$ so that the consistency is maintained.

The discovery of the 249 lines through $(0,0)$ may be done by hand or by simple mechanical sorting procedures. To check and to facilitate the consideration of other possible structures a program for the automatic computer $S W A C$ has been written which will determine the lines consistent with any square which is the table of a loop.

The problem of the search is now to find sets of 63 additional lines from the 2241 such that each resulting total of 90 lines contains exactly one line joining any two distinct points, $(a, b)$ and $(c, d)$. For $a=c$ or $b=d$ we have the line $x=a=c$ or $y=b=d$ in one of the pre-determined pencils.

The search described would be far too extensive in practice and a procedure must be found to reduce the total number of sets to be looked for and the total number of isomorphic planes to be found. To do this we consider automorphisms which preserve the 27 fixed lines of the three basic pencils. The group $G$ which takes the three pencils into themselves consists of 81.48 automorphisms. Of these 81 translations $T$ have the form:

$$
(x, y) \rightarrow(x+a, y+b) \text {. }
$$

The subgroup $H$ fixing $(0,0)$ has 48 elements and is essentially the automorphism group of the elementary abelian group of order 9 . These automorphisms are of the form:

$$
(x, y) \rightarrow(\alpha(x), \alpha(y))
$$

where $\alpha$ is a permutation of $0,1, \cdots, 8$ which leaves 0 fixed. This group contains a subgroup $U$ of order 6 . The permutations $\alpha$ of $U$ are:

$$
\begin{aligned}
& \alpha_{1}=I \\
& \alpha_{2}=(345)(687) \\
& \alpha_{3}=(354)(678) \\
& \alpha_{4}=(36)(47)(58) \\
& \alpha_{5}=(37)(48)(56) \\
& \alpha_{6}=(38)(46)(57)
\end{aligned}
$$

The group $H$ may now be given by

$$
H=U+U \alpha_{7}+U \alpha_{13}+U \alpha_{19}+U \alpha_{25}+U \alpha_{31}+U \alpha_{37}+U \alpha_{48}
$$

where

$$
\begin{aligned}
& \alpha_{7}=(12)(36)(48)(57) \\
& \alpha_{13}=(13472685) \\
& \alpha_{19}=(1428)(3765) \\
& \alpha_{25}=(15862743) \\
& \alpha_{31}=(16452387) \\
& \alpha_{37}=(17832546) \\
& \alpha_{43}=(1824)(3567) .
\end{aligned}
$$


To apply the automorphisms $T$ of 2.3 to a line $L$ the product $A_{a}{ }^{-1} L A_{b}$ is computed where $A_{a}$ and $A_{b}$ are the permutations of (2.1) containing taking 0 to $a$ and $b$ respectively. To apply an automorphism $\alpha$ of $H$ to $L, \alpha^{-1} L \alpha$ is calculated. As these automorphisms leave the lines (2.1) invariant they take consistent lines to consistent lines and this fact may be used in the construction and checking of the consistent lines. The discussion of the method by which the 2241 lines are obtained from 249 may be simply phrased in terms of translations.

There are still other automorphisms which exchange the pencils $x=c$ (through $(\infty)$ on $\left.L_{\infty}\right), y=c$ (through $(0)$ on $\left.L_{\infty}\right)$ and $y=x+c$ (through (1) on $L_{\infty}$ ) among themselves. The reflection $R$ about $y=x$ is given by

$$
(x, y)=(y, x) \text {. }
$$

This is applied to $L$ by computing $L^{-1}$.

There is also the automorphism $C$ :

$$
(x, y) \rightarrow(x, x-y) .
$$

$C$ and $R$ together generate a group $K$ of order 6 which permutes $(\infty),(0)$ and (1) in all possible ways.

3. Classification of consistent lines. The 249 lines which pass through $(0,0)$ and are consistent with (2.1) are interchanged by the group $H$. These lines fall into 12 equivalence classes with respect to this group.

We list here one representative of each class and the number of lines in each class.

\begin{tabular}{ccccccccccc} 
Class & \multicolumn{11}{c}{ Line } & & \multicolumn{4}{c}{$\begin{array}{c}\text { Number of } \\
\text { Conjugates }\end{array}$} \\
1 & 0 & 2 & 1 & 6 & 8 & 7 & 3 & 5 & 4 & 1 \\
2 & 0 & 2 & 1 & 6 & 8 & 7 & 4 & 3 & 5 & 16 \\
3 & 0 & 2 & 1 & 7 & 6 & 8 & 4 & 3 & 5 & 8 \\
4 & 0 & 2 & 1 & 7 & 6 & 8 & 5 & 4 & 3 & 8 \\
5 & 0 & 2 & 3 & 1 & 6 & 8 & 5 & 4 & 7 & 48 \\
6 & 0 & 2 & 3 & 8 & 7 & 1 & 4 & 6 & 5 & 48 \\
7 & 0 & 3 & 5 & 2 & 1 & 6 & 4 & 8 & 7 & 48 \\
8 & 0 & 3 & 6 & 2 & 5 & 8 & 1 & 4 & 7 & 6 \\
9 & 0 & 3 & 6 & 2 & 7 & 4 & 1 & 8 & 5 & 6 \\
10 & 0 & 3 & 6 & 4 & 1 & 8 & 5 & 2 & 7 & 48 \\
11 & 0 & 3 & 6 & 4 & 7 & 1 & 8 & 2 & 5 & 6 \\
12 & 0 & 3 & 6 & 7 & 1 & 4 & 5 & 8 & 2 & 6 \\
& & & & & & & & & Total & 249
\end{tabular}

The distribution of these lines may be represented as follows:

Form of line

Class

$\begin{array}{lrrrrrrrrrrrr} & 1 & 2 & 3 & 4 & 5 & 6 & 7 & 8 & 9 & 10 & 11 & 12 \\ 02 \cdots & 1 & 10 & 2 & 2 & 6 & 6 & 0 & 0 & 0 & 0 & 0 & 0 \\ 0 i \cdots(i=3, \cdots, 8) & 0 & 1 & 1 & 1 & 7 & 7 & 8 & 1 & 1 & 8 & 1 & 1\end{array}$

The classes tabulated in (3.1) and (3.2) are equivalences under $H$ alone. With respect to $G$, obviously each of the 2241 lines is equivalent to one of the lines of 
(3.1) but some of the classes may be further amalgamated. For example, let $L_{2}$ be the representative of class 2 in (3.1); if we apply to $L_{2}$ first the translation $(x, y) \rightarrow$ $(x+3, y+8)$ and then the automorphism $\alpha_{7}=(12)(36)(48)(57)$ of $H$, the repre sentative of class 3 results:

$$
\begin{aligned}
& A_{3}^{-1} L_{2} A_{8}=A_{6} L_{2} A_{8}=(063)(174)(285) \cdot(12)(364857)(084)(165)(273) \\
&=(12)(38)(47)(56) \\
&(12)(36)(48)(57)(12)(38)(47)(56)(12)(36)(48)(57)=(12)(37)(46)(58)=L_{3} .
\end{aligned}
$$

In a similar manner the classes $5,6,7$ amalgamate under $G$. Specifically if we denote the elements of $H:(12)(34)(68)$ and (12)(274658), by $\alpha_{12}$ and $\alpha_{9}$ respectively and follow the rule of denoting the line (permutation) representing the $i$ th class of (3.1) by $L_{i}$ we have:

and

$$
\alpha_{12} A_{2}^{-1} L_{5} A_{1} \alpha_{12}=L_{6}
$$

$$
\alpha_{9}{ }^{-1} A_{1}^{-1} L_{5} A_{6} \alpha_{9}=L_{7} .
$$

Further, classes 9 and 10 amalgamate under $G$ but classes 1, 4, 8, 11, 12 remain distinct. However, using the group $K$ which permutes the basic pencils, we find that classes 8 and 12 are equivalent under the transformation $C$ while 11 and 12 are equivalent under $R$. Thus classes $8,11,12$ amalgamate under the group $K$. These are the only new equivalences provided by $K$ and there finally remain 6 classes: 1 ; $(2,3) ; 4 ;(5,6,7) ;(8,11,12) ;(9,10)$.

Although many of the procedures discussed in this section are essentially mechanical in nature, programming is at least as difficult as the hand computations and the latter method was utilized for all these results.

4. Construction of pencils through $(0,0)$. A plane $\pi$ containing the additive pencil $(2.1)$ must contain a line through $(0,0)$ and $(1,2)$, that is a line whose representation is of the form $02 \cdots$. From (3.2) it is clear that such a line must belong to one of the classes $1-6$. But, using the equivalences deduced under $G$, we may assume that it belongs to one of the classes $1,3,4,6$ since a line of class 2 is equivalent to one of class 3 and a line of class 5 will be transformed to one of class 6 .

Among these four classes we shall establish a preference order or hierarchy in the order 1, 6, 4, 3. That is, we shall eliminate all lines of class 1 from consideration when making a search using a class 6 line; again when using a class 4 line we shall eliminate any line of class $1,5,6$, or 7 ; finally using a class 3 line for a start any line of classes, $1,4,5,6$, and 7 is excluded. This preference order helps to eliminate duplications since each plane will appear only with its most preferred line.

The first stage of the search consists in finding, for each start, the possible pencils through $(0,0)$. When $A_{0}$ of $(2.1)$ is eliminated and we ignore the first column, such a pencil is represented by a latin square of order 8 on the numbers $1,2, \cdots, 8$ whose first line is the designated start and whose subsequent lines are selected from the 249 permissible lines through $(0,0)$ with due respect for preference order.

A considerable portion of this search was carried out by hand. The complete 
search was made independently by SWAC and the results compared for the duplicated parts.

The mechanical procedure first finds for each of the four starts, the lines through $(0,0),(1, i), i=3, \cdots, 8$ consistent with the start; a further rejection is then made on the basis of preference. Next, a particular line through $(0,0),(1,3)$ is selected and all remaining lines consistent with it are used in the high speed memory of SWAC to form by a continuation of these processes all possible latin squares. Then the next line through $(1,3)$ is taken and so on to completion.

With the set of possible pencils before us, we may proceed in either of two ways (or a combination of them). We may eliminate equivalent pencils on the basis of the automorphism groups $H$ and $K$ which leave $(0,0)$ fixed, and attempt to complete the surviving representatives to planes or we may eliminate the pencils which cannot be completed and then discuss possible equivalences among the survivors. The first choice is better adapted to hand methods, the second to machines.

In the cases which produced a large number of possible pencils it was desirable to introduce a secondary preference order. In particular, the class 1 line, $L_{1}$, goes into itself under all substitutions of $H$; moreover lines through $(0,0)$ in classes 2 , $3,4,5$ and 6 are not consistent with $L_{1}$. Hence any line through $(0,0)$ consistent with $L_{1}$ may be carried by elements of $H$ into one of $L_{7}, L_{10}, L_{8}, L_{9}, L_{11}, L_{12}$. These, in this order, were adopted as secondary preferences and the total output of squares of type 1 was thus reduced to 50 .

Under the automorphisms of $H \cdot K$ these pencils fall into 17 classes. Similarly, the class 6 start produced 57 pencils ln 11 classes; the class 4 start 12 pencils in 3 classes. No pencils are found in class 3 .

In the mechanical procedure, the 249 possible lines through a point $(x, 0)$ are first found by translating the 249 lines through $(0,0)$. Each of these lines is then compared with a square and those lines which intersect no line of the square in more than one point are stored and eventually tabulated with the square. It frequently occurred that in the tabulation for a particular square no line through $(0, y)$ was present for at least one $y$. This square could be at once rejected as impossible to complete.

In all routines used on SWAC the major operation was one of sorting. To facilitate this sorting, positional coding was used. Each element $y_{i}$ of a line was assigned eight consecutive spaces in a register (since $S W A C$ uses 36 bit words, double precision was required to store a full line). The number $i$ is represented by a 1 in the $i$ th space and 0 's in the other 7; 0 is represented by 0 's in all eight places. Using complementation and extraction, the number of intersections was representable as the number of 1 bits in a resulting word pair.

5. Representative pencils which are completable to a plane. Most of the squares found cannot be completed; further, most of the squares which do lead to planes do so uniquely. After a few additional pencils are added from the tabulations described above the remainder of the lines are essentially decided and it is a simple matter to write them down.

When, in either order, the equivalences of pencils through $(0,0)$ have been taken into account and completability determined, we find 12 pencils remaining, 8 of class 
1, 1 of class 6 and 3 of class 4 . These are tabulated here omitting the lines $x=0$, $y=0, x=y$ common to all cases. The numbering corresponds to the original position of the square in the original list of squares mentioned above.

Class 1 representative pencils which can be completed

$\begin{array}{lllllllll}0 & 2 & 1 & 6 & \mathbf{8} & \mathbf{7} & 3 & 5 & 4 \\ 0 & 3 & 5 & 2 & 1 & 6 & 4 & 8 & 7 \\ 0 & 4 & 3 & 8 & 2 & 1 & 7 & 6 & 5 \\ 0 & 5 & 4 & 1 & 7 & 2 & 8 & 3 & 6 \\ 0 & 6 & 8 & 7 & 5 & 4 & 2 & 1 & 3 \\ 0 & 7 & 6 & 4 & 3 & 8 & 5 & 2 & 1 \\ 0 & 8 & 7 & 5 & 6 & 3 & 1 & 4 & 2\end{array}$

$\begin{array}{lllllllll}0 & 2 & 1 & 6 & 8 & 7 & 3 & 5 & 4 \\ 0 & 3 & 6 & 4 & 1 & 8 & 5 & 2 & 7 \\ 0 & 4 & 7 & 1 & 3 & 6 & 2 & 8 & 5 \\ 0 & 5 & 8 & 2 & 7 & 3 & 4 & 6 & 1 \\ 0 & 6 & 4 & 7 & 5 & 2 & 8 & 1 & 3 \\ 0 & 7 & 5 & 8 & 2 & 4 & 1 & 3 & 6 \\ 0 & 8 & 3 & 5 & 6 & 1 & 7 & 4 & 2\end{array}$

$\begin{array}{lllllllll}0 & 2 & 1 & 6 & 8 & 7 & 3 & 5 & 4\end{array}$

$\begin{array}{lllllllll}0 & 2 & 1 & 6 & 8 & 7 & 3 & 5 & 4\end{array}$

$\begin{array}{lllllllll}0 & 3 & 6 & 4 & 1 & 8 & 5 & 2 & 7\end{array}$

$\begin{array}{lllllllll}0 & 3 & 6 & 4 & 1 & 8 & 5 & 2 & 7\end{array}$

$\begin{array}{lllllllll}0 & 4 & 7 & 1 & 3 & 6 & 2 & 8 & 5\end{array}$

(1.28)

$\begin{array}{lllllllll}0 & 4 & 7 & 5 & 6 & 2 & 1 & 8 & 3\end{array}$

$\begin{array}{lllllllll}0 & 5 & 8 & 2 & 6 & 4 & 7 & 1 & 3\end{array}$

$\begin{array}{lllllllll}0 & 6 & 5 & 7 & 2 & 3 & 8 & 4 & 1\end{array}$

$\begin{array}{lllllllll}0 & 5 & 8 & 1 & 7 & 4 & 2 & 3 & 6\end{array}$

$\begin{array}{lllllllll}0 & 7 & 3 & 8 & 5 & 1 & 4 & 6 & 2\end{array}$

$\begin{array}{lllllllll}0 & 6 & 5 & 7 & 2 & 3 & 8 & 4 & 1\end{array}$

$\begin{array}{lllllllll}0 & 8 & 4 & 5 & 7 & 2 & 1 & 3 & 6\end{array}$

$\begin{array}{lllllllll}0 & 7 & 3 & 8 & 5 & 1 & 4 & 6 & 2\end{array}$

$\begin{array}{lllllllll}0 & 2 & 1 & 6 & 8 & 7 & 3 & 5 & 4\end{array}$

$\begin{array}{lllllllll}0 & 8 & 4 & 2 & 3 & 6 & 7 & 1 & 5\end{array}$

$\begin{array}{lllllllll}0 & 3 & 6 & 4 & 1 & 8 & 5 & 2 & 7\end{array}$

$\begin{array}{lllllllll}0 & 2 & 1 & 6 & 8 & 7 & 3 & 5 & 4\end{array}$

$\begin{array}{lllllllll}0 & 4 & 7 & 8 & 2 & 3 & 1 & 6 & 5\end{array}$

$\begin{array}{llllllllll}(1.30) & 0 & 5 & 8 & 1 & 7 & 4 & 2 & 3 & 6\end{array}$

$\begin{array}{lllllllll}0 & 6 & 4 & 7 & 5 & 2 & 8 & 1 & 3\end{array}$

(1.36)

$\begin{array}{lllllllll}0 & 3 & 6 & 2 & 5 & 8 & 1 & 4 & 7\end{array}$

$\begin{array}{lllllllll}0 & 7 & 5 & 2 & 3 & 6 & 4 & 8 & 1\end{array}$

$\begin{array}{lllllllll}0 & 4 & 8 & 7 & 2 & 3 & 5 & 6 & 1\end{array}$

$\begin{array}{lllllllll}0 & 8 & 3 & 5 & 6 & 1 & 7 & 4 & 2\end{array}$

$\begin{array}{lllllllll}0 & 5 & 7 & 4 & 6 & 2 & 8 & 1 & 3\end{array}$

$\begin{array}{lllllllll}0 & 6 & 3 & 1 & 7 & 4 & 2 & 8 & 5\end{array}$

$\begin{array}{lllllllll}0 & 7 & 5 & 8 & 3 & 1 & 4 & 2 & 6\end{array}$

$\begin{array}{lllllllll}0 & 8 & 4 & 5 & 1 & 6 & 7 & 3 & 2\end{array}$

$\begin{array}{lllllllll}0 & 2 & 1 & 6 & 8 & 7 & 3 & 5 & 4\end{array}$

$\begin{array}{lllllllll}0 & 2 & 1 & 6 & 8 & 7 & 3 & 5 & 4\end{array}$

$\begin{array}{lllllllll}0 & 3 & 6 & 2 & 5 & 8 & 1 & 4 & 7\end{array}$

$\begin{array}{lllllllll}0 & 3 & 6 & 2 & 7 & 4 & 1 & 8 & 5\end{array}$

$\begin{array}{lllllllll}0 & 4 & 8 & 5 & 6 & 1 & 7 & 2 & 3\end{array}$

$\begin{array}{llllllllll}(1.35) & 0 & 5 & 7 & 8 & 1 & 3 & 4 & 6 & 2\end{array}$

$\begin{array}{lllllllll}0 & 6 & 3 & 1 & 7 & 4 & 2 & 8 & 5\end{array}$

$\begin{array}{lllllllll}0 & 4 & 8 & 5 & 2 & 6 & 7 & 3 & 1\end{array}$

$\begin{array}{llllllllll}(1.44) & 0 & 5 & 7 & 8 & 3 & 2 & 4 & 1 & 6\end{array}$

$\begin{array}{lllllllll}0 & 6 & 3 & 1 & 5 & 8 & 2 & 4 & 7\end{array}$

$\begin{array}{lllllllll}0 & 7 & 5 & 4 & 2 & 6 & 8 & 3 & 1\end{array}$

$\begin{array}{lllllllll}0 & 7 & 5 & 4 & 6 & 1 & 8 & 2 & 3\end{array}$

$\begin{array}{lllllllll}0 & 8 & 4 & 7 & 3 & 2 & 5 & 1 & 6\end{array}$

$\begin{array}{lllllllll}0 & 8 & 4 & 7 & 1 & 3 & 5 & 6 & 2\end{array}$

Class 6 representative pencil which can be completed

$\begin{array}{rlllllllll} & 0 & 2 & 3 & 8 & 7 & 1 & 4 & 6 & 5 \\ & 0 & 3 & 6 & 4 & 8 & 2 & 5 & 1 & 7 \\ & 0 & 4 & 7 & 5 & 1 & 6 & 2 & 8 & 3 \\ (6.17) & 0 & 5 & 8 & 1 & 6 & 4 & 7 & 3 & 2 \\ & 0 & 6 & 5 & 7 & 2 & 3 & 8 & 4 & 1 \\ 0 & 7 & 4 & 2 & 3 & 8 & 1 & 5 & 6 \\ 0 & 8 & 1 & 6 & 5 & 7 & 3 & 2 & 4\end{array}$


Class 4 representative pencils which can be completed

$\begin{array}{lllllllllllllllllll}0 & 2 & 1 & 7 & 6 & 8 & 5 & 4 & 3 & & 0 & 2 & 1 & 7 & 6 & 8 & 5 & 4 & 3 \\ 0 & 3 & 6 & 2 & 7 & 4 & 1 & 8 & 5 & & 0 & 3 & 6 & 4 & 7 & 1 & 8 & 2 & 5 \\ 0 & 4 & 8 & 5 & 2 & 6 & 7 & 3 & 1 & & 0 & 4 & 8 & 2 & 3 & 7 & 1 & 5 & 6 \\ 0 & 5 & 7 & 8 & 3 & 2 & 4 & 1 & 6 & (4.4) & 0 & 5 & 7 & 6 & 2 & 4 & 3 & 8 & 1 \\ 0 & 6 & 3 & 4 & 1 & 7 & 8 & 5 & 2 & & 0 & 6 & 3 & 8 & 5 & 2 & 4 & 1 & 7 \\ 0 & 7 & 5 & 1 & 8 & 3 & 2 & 6 & 4 & & 0 & 7 & 5 & 1 & 8 & 3 & 2 & 6 & 4 \\ 0 & 8 & 4 & 6 & 5 & 1 & 3 & 2 & 7 & 0 & 8 & 4 & 5 & 1 & 6 & 7 & 3 & 2\end{array}$

$$
\begin{aligned}
& \begin{array}{lllllllll}
0 & 2 & 1 & 7 & 6 & 8 & 5 & 4 & 3
\end{array} \\
& \begin{array}{lllllllll}
0 & 3 & 6 & 5 & 8 & 2 & 7 & 1 & 4
\end{array} \\
& \begin{array}{lllllllll}
0 & 4 & 7 & 1 & 3 & 6 & 2 & 8 & 5
\end{array} \\
& \begin{array}{llllllllll}
(4.5) & 0 & 5 & 8 & 2 & 7 & 3 & 4 & 6 & 1
\end{array} \\
& \begin{array}{lllllllll}
0 & 6 & 3 & 4 & 1 & 7 & 8 & 5 & 2
\end{array} \\
& \begin{array}{lllllllll}
0 & 7 & 5 & 8 & 2 & 4 & 1 & 3 & 6
\end{array} \\
& \begin{array}{lllllllll}
0 & 8 & 4 & 6 & 5 & 1 & 3 & 2 & 7
\end{array}
\end{aligned}
$$

Each of the 12 has a unique completion to a full plane except 1.44 which has nine completions. But, using our preference order, the class 6 plane may be removed from further consideration. In the completion of the 6.17 square the class 1 line, 354021687 , occurs unequivocally.

The tabulations yield 8 possible pencils through $(2,0)$ consistent with 1.44 . With one exception each of these is uniquely completable; the first has two completions. These pencils are listed here, omitting the lines $x=2, y=0$ and $y=x+1$.

$$
(2,0) \text { pencils for } 1.44
$$

$\begin{array}{lllllllll}2 & 1 & 0 & 8 & 7 & 6 & 5 & 4 & 3 \\ 3 & 6 & 0 & 7 & 4 & 2 & 8 & 5 & 1 \\ 4 & 8 & 0 & 2 & 6 & 5 & 3 & 1 & 7\end{array}$

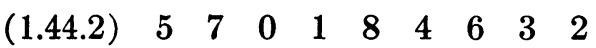

$\begin{array}{lllllllll}5 & 7 & 0 & 3 & 2 & 8 & 1 & 6 & 4\end{array}$

$\begin{array}{lllllllll}6 & 3 & 0 & 5 & 8 & 1 & 4 & 7 & 2\end{array}$

$\begin{array}{lllllllll}7 & 5 & 0 & 6 & 1 & 4 & 2 & 3 & 8\end{array}$

$\begin{array}{lllllllll}8 & 4 & 0 & 1 & 3 & 7 & 6 & 2 & 5\end{array}$

$\begin{array}{lllllllll}2 & 1 & 0 & 8 & 7 & 6 & 5 & 4 & 3\end{array}$

$\begin{array}{lllllllll}3 & 6 & 0 & 5 & 1 & 7 & 4 & 2 & 8\end{array}$

$\begin{array}{lllllllll}4 & 8 & 0 & 6 & 3 & 1 & 2 & 7 & 5\end{array}$

$\begin{array}{llllllllll}(1.44 .2) & 5 & 7 & 0 & 1 & 8 & 4 & 6 & 3 & 2 \\ & 6 & 3 & 0 & 7 & 2 & 5 & 8 & 1 & 4\end{array}$

$\begin{array}{lllllllll}7 & 5 & 0 & 2 & 4 & 8 & 3 & 6 & 1\end{array}$

$\begin{array}{lllllllll}8 & 4 & 0 & 3 & 6 & 2 & 1 & 5 & 7\end{array}$

$\begin{array}{lllllllll}2 & 1 & 0 & 8 & 7 & 6 & 5 & 4 & 3\end{array}$

$\begin{array}{lllllllll}3 & 7 & 0 & 2 & 8 & 5 & 4 & 6 & 1\end{array}$

$\begin{array}{llllllllll}(1.44 .3) & 5 & 8 & 0 & 6 & 4 & 2 & 1 & 3 & 7\end{array}$

$\begin{array}{lllllllll}6 & 4 & 0 & 7 & 3 & 1 & 2 & 5 & 8\end{array}$

$\begin{array}{lllllllll}7 & 3 & 0 & 5 & 1 & 8 & 6 & 2 & 4\end{array}$

$\begin{array}{lllllllll}8 & 5 & 0 & 1 & 6 & 4 & 3 & 7 & 2\end{array}$ $\begin{array}{lllllllll}2 & 1 & 0 & 8 & 7 & 6 & 5 & 4 & 3\end{array}$

$\begin{array}{lllllllll}3 & 6 & 0 & 5 & 1 & 7 & 4 & 2 & 8\end{array}$

$\begin{array}{lllllllll}4 & 7 & 0 & 2 & 3 & 8 & 6 & 5 & 1\end{array}$

$\begin{array}{llllllllll}(1.44 .5) & 5 & 8 & 0 & 6 & 4 & 2 & 1 & 3 & 7\end{array}$

$\begin{array}{lllllllll}6 & 3 & 0 & 7 & 2 & 5 & 8 & 1 & 4\end{array}$

$\begin{array}{lllllllll}7 & 4 & 0 & 3 & 8 & 1 & 2 & 6 & 5\end{array}$

$\begin{array}{lllllllll}8 & 5 & 0 & 1 & 6 & 4 & 3 & 7 & 2\end{array}$

$\begin{array}{lllllllll}2 & 1 & 0 & 7 & 6 & 8 & 4 & 3 & 5\end{array}$

$\begin{array}{lllllllll}3 & 7 & 0 & 8 & 2 & 5 & 1 & 6 & 4\end{array}$

$\begin{array}{lllllllll}4 & 6 & 0 & 3 & 7 & 2 & 8 & 5 & 1\end{array}$

$\begin{array}{llllllllll}(1.44 .6) & 5 & 8 & 0 & 2 & 4 & 6 & 3 & 1 & 7\end{array}$

$\begin{array}{lllllllll}6 & 4 & 0 & 1 & 3 & 7 & 5 & 2 & 8\end{array}$

$\begin{array}{lllllllll}7 & 3 & 0 & 5 & 8 & 1 & 6 & 4 & 2\end{array}$

$\begin{array}{lllllllll}8 & 5 & 0 & 6 & 1 & 4 & 2 & 7 & 3\end{array}$

$\begin{array}{lllllllll}2 & 1 & 0 & 8 & 7 & 6 & 5 & 4 & 3\end{array}$

$\begin{array}{lllllllll}3 & 7 & 0 & 2 & 8 & 5 & 4 & 6 & 1\end{array}$

$\begin{array}{lllllllll}4 & 8 & 0 & 6 & 3 & 1 & 2 & 7 & 5\end{array}$

$\begin{array}{llllllllll}(1.44 .7) & 5 & 6 & 0 & 7 & 2 & 4 & 3 & 1 & 8\end{array}$

$\begin{array}{lllllllll}6 & 5 & 0 & 1 & 4 & 7 & 8 & 3 & 2\end{array}$

$\begin{array}{lllllllll}7 & 3 & 0 & 5 & 1 & 8 & 6 & 2 & 4\end{array}$

$\begin{array}{lllllllll}8 & 4 & 0 & 3 & 6 & 2 & 1 & 5 & 7\end{array}$ 
$(2,0)$ pencils for 1.44 -continued

\begin{tabular}{lllllllll}
\multicolumn{10}{c}{$(2,0)$} & pencils for \\
2 & 1 & 0 & 8 & 7 & 6 & 5 & 4 & 3 \\
3 & 8 & 0 & 5 & 6 & 2 & 1 & 7 & 4 \\
4 & 7 & 0 & 2 & 3 & 8 & 6 & 5 & 1 \\
5 & 6 & 0 & 7 & 2 & 4 & 3 & 1 & 8 \\
6 & 5 & 0 & 1 & 4 & 7 & 8 & 3 & 2 \\
7 & 4 & 0 & 3 & 8 & 1 & 2 & 6 & 5 \\
8 & 3 & 0 & 6 & 1 & 5 & 4 & 2 & 7
\end{tabular}

$\begin{array}{llllllllll} & 2 & 1 & 0 & 8 & 7 & 6 & 5 & 4 & 3 \\ 3 & 8 & 0 & 5 & 6 & 2 & 1 & 7 & 4 \\ 4 & 6 & 0 & 3 & 2 & 7 & 8 & 1 & 5 \\ (1.44 .8) & 5 & 7 & 0 & 1 & 8 & 4 & 6 & 3 & 2 \\ 6 & 4 & 0 & 7 & 3 & 1 & 2 & 5 & 8 \\ 7 & 5 & 0 & 2 & 4 & 8 & 3 & 6 & 1 \\ 8 & 3 & 0 & 6 & 1 & 5 & 4 & 2 & 7\end{array}$

To give a final separation of the completable planes it suffices to indicate the pencils through $(3,0)$ for 1.44.1. Again lines $x=3, y=0, y=x+6$ are omitted:

$$
(3,0) \text { pencils for } 1.44 .1
$$

\begin{tabular}{|c|c|c|c|c|c|c|c|c|c|c|c|c|c|c|c|c|c|}
\hline & 1 & 4 & 7 & 0 & 8 & 5 & 2 & 6 & 3 & 1 & 8 & 5 & $\mathbf{0}$ & 3 & 6 & 2 & 7 \\
\hline & 2 & 8 & 5 & 0 & 4 & 7 & 1 & 3 & 6 & 2 & 4 & 7 & 0 & 6 & 3 & 1 & 5 \\
\hline & 3 & 5 & 4 & 0 & 2 & 1 & 6 & 8 & 7 & 3 & 5 & 4 & 0 & 2 & 1 & 6 & 8 \\
\hline$(1.44 .1 .1)$ & 4 & 2 & 6 & 0 & 3 & 8 & 5 & 7 & $1(1.44 .1 .2)$ & 4 & 1 & 6 & 0 & 5 & 7 & 8 & 3 \\
\hline & 5 & 6 & 1 & 0 & 7 & 3 & 4 & 2 & 8 & 5 & 6 & 2 & 0 & 8 & 4 & 7 & 1 \\
\hline & 7 & 3 & 2 & 0 & 5 & 6 & 8 & 1 & 4 & 7 & 3 & 1 & 0 & 4 & 8 & 5 & 2 \\
\hline & 8 & 1 & 3 & 0 & 6 & 4 & 7 & 5 & 2 & 8 & 2 & 3 & 0 & 7 & 5 & 4 & 6 \\
\hline
\end{tabular}

6. Equivalence of completed planes. It remains to recognize the distinct planes among the 19 completed planes (excluding that of class 6). For the purposes of tabulation we distinguish the four previously known planes as follows:

A. The desarguesian plane-coordinatizable by GF(9); this appears as 1.35 , this pencil through $(0,0)$ being readily recognizable as giving the multiplication table of the field.

B. The Veblen-Wedderburn plane-coordinatizable by a near field. Again this multiplication may be recognized in the square given for 1.36.

C. The dual Veblen-Wedderburn plane-This may be exhibited as the plane constructable from 1.44.2.

D. The plane whose coordinatization in terms of an elementary abelian addition was indicated by Hughes [3]. This appears in 1.44.1.1.

Of these, planes A, B, C have lines of the form $y=x m+b$ in their standard form and, using the additive pencil (2.1) and the multiplicative pencil through $(0,0)$ tabulated in the last section, the remaining lines may easily be added by the reader who wishes a complete tabulation. For B the distributive law $\left(x_{1}+x_{2}\right) m=x_{1} m+$ $x_{2} m$ holds; the multiplications are automorphisms of the additive group. For $\mathrm{C}$ the distributive law $x\left(m_{1}+m_{2}\right)=x m_{1}+x m_{2}$ is valid.

For $\mathrm{D}$ we give the complete tabulation of 1.44 .1 .1 below. In order that the reader may follow the details of the arguments on equivalence of planes we also list the completions of 1.44.1.2, 1.44.6, 1.4 and 4.3.

We now maintain that the nineteen planes may be classified as follows:
A. 1.35
B. $1.36,1.4,1.44 .6,4.4$
C. $1.44 .2,1.44 .1 .2,1.44 .3,1.44 .4,1.27,1.28$
D. $1.44 .1 .1,1.44 .5,1.44 .7,1.44 .8,1.26,1.30,4.3,4.5$.

To begin with class B, 4.4 is the Veblen-Wedderburn plane coordinatized by the system in which -1 is not in the center. This system is designated by the letter 
$U$ in [1]; as shown in this reference (p. 274) the same plane results as that produced by the nearfield. The translation $L \rightarrow A_{1}^{-1} L A_{2}$ or $(x, y) \rightarrow(x+1, y+2)$ takes 1.4 into 1.44.6. It is somewhat more difficult to show the equivalence of 1.44 .6 and 1.36. In the projective completion of 1.44 .6 we make the following replacements.

$$
\begin{aligned}
&(0,0) \rightarrow(\infty) \\
&(\infty) \rightarrow(0,0) \\
&(0) \rightarrow(0) \\
&(1,1) \rightarrow(1,1) \\
&(\infty)\left(\begin{array}{lllllllllll}
1 & 1 & 1 & 1 & 1 & 1 & 1 & 1 & 1 \\
0 & 1 & 2 & 3 & 4 & 5 & 6 & 7 & 8
\end{array}\right) \rightarrow\left(\begin{array}{llllllll}
0 \\
0
\end{array}\right)(1)\left(\begin{array}{llllllll}
1 & 2 & 3 & 4 & 5 & 6 & 7 & 8 \\
1 & 2 & 3 & 4 & 5 & 6 & 7 & 8
\end{array}\right)
\end{aligned}
$$

where the last replacement indicates that the line $x=1$ is replaced by the line $x=y$ in the manner shown. With the new coordinates 1.44 .6 is transformed to 1.36 . This establishes the equivalences under $\mathrm{B}$.

For C, the translation $L \rightarrow A_{1}^{-1} L A_{3}$ takes 1.27 to 1.44 .3 and 1.28 into 1.44.4. The transformation $L \rightarrow L^{-1}$ interchanges 1.44 .2 and 1.44.1.2. The automorphism $C+(x, y) \rightarrow(x, x-y)$ interchanges 1.44 .4 and 1.44.1.2. Finally the mapping $L \rightarrow L(12)(36)(48)(57)$ interchanges 1.44 .3 and 1.44.4. This combination shows the equivalence of the planes in class $\mathrm{C}$.

In class $D, L \rightarrow A_{1}{ }^{-1} L A_{3}$ takes 1.26 to 1.44 .8 and 1.30 to 1.44 .7 . The mapping $L \rightarrow(06)(17)(28) L(012)(375648)$ takes 4.5 into 4.3 . We introduce new coordinates into the projective completion of 4.3 by making the replacements:

$$
\begin{aligned}
&(2) \rightarrow(\infty) \\
&(6) \rightarrow(0) \\
&(0,0) \rightarrow(0,0) \\
&(8,4) \rightarrow(1,1) \\
& \text { (7) }\left(\begin{array}{lllllllllllllll}
0 & 1 & 2 & 3 & 4 & 5 & 6 & 7 & 8 \\
0 & 7 & 5 & 1 & 8 & 3 & 2 & 6 & 4
\end{array}\right) \rightarrow(1)\left(\begin{array}{lllllllll}
0 & 7 & 5 & 4 & 2 & 6 & 8 & 3 & 1 \\
0 & 7 & 5 & 4 & 2 & 6 & 8 & 3 & 1
\end{array}\right) .
\end{aligned}
$$

Under this change the plane 4.3 is found to be identical with plane 1.44.1.1. The transformation $L \rightarrow(354)(678) L(345)(687)$ takes 1.44 .8 into 1.44.5. Also $L \rightarrow$ (345) (687) $L(354)(678)$ takes 1.44 .7 into 1.44.5. Finally, $L \rightarrow(13472685) L(1586$ 2743) takes 1.44.5 into 1.44.1.1. This completes the tables of equivalences.

Plane 1.44.1.1

$\begin{array}{llllllllll}* & 0 & 1 & 2 & 3 & 4 & 5 & 6 & 7 & 8 \\ * & 1 & 2 & 0 & 4 & 5 & 3 & 7 & 8 & 6 \\ * & 2 & 0 & 1 & 5 & 3 & 4 & 8 & 6 & 7 \\ * & 3 & 4 & 5 & 6 & 7 & 8 & 0 & 1 & 2 \\ * & 4 & 5 & 3 & 7 & 8 & 6 & 1 & 2 & 0 \\ * & 5 & 3 & 4 & 8 & 6 & 7 & 2 & 0 & 1 \\ * & 6 & 7 & 8 & 0 & 1 & 2 & 3 & 4 & 5 \\ * & 7 & 8 & 6 & 1 & 2 & 0 & 4 & 5 & 3 \\ * & 8 & 6 & 7 & 2 & 0 & 1 & 5 & 3 & 4\end{array}$

$\begin{array}{llllllllll}* & 0 & 2 & 1 & 6 & 8 & 7 & 3 & 5 & 4 \\ * & 1 & 0 & 2 & 7 & 6 & 8 & 4 & 3 & 5 \\ * & 2 & 1 & 0 & 8 & 7 & 6 & 5 & 4 & 3 \\ * & 3 & 5 & 4 & 0 & 2 & 1 & 6 & 8 & 7 \\ * & 4 & 3 & 5 & 1 & 0 & 2 & 7 & 6 & 8 \\ * & 5 & 4 & 3 & 2 & 1 & 0 & 8 & 7 & 6 \\ * & 6 & 8 & 7 & 3 & 5 & 4 & 0 & 2 & 1 \\ * & 7 & 6 & 8 & 4 & 3 & 5 & 1 & 0 & 2 \\ * & 8 & 7 & 6 & 5 & 4 & 3 & 2 & 1 & 0\end{array}$

Note: Starred lines are common to 1.44.1.1 and 1.44.1.2. 
Plane 1.44.1.1-continued

$\begin{array}{llllllllll}* & 0 & 3 & 6 & 2 & 7 & 4 & 1 & 8 & 5\end{array}$ $\begin{array}{lllllllll}1 & 4 & 7 & 0 & 8 & 5 & 2 & 6 & 3\end{array}$ $\begin{array}{lllllllll}2 & 5 & 8 & 1 & 6 & 3 & 0 & 7 & 4\end{array}$

* $3 \begin{array}{lllllllll}6 & 6 & 0 & 7 & 4 & 2 & 8 & 5 & 1\end{array}$ $\begin{array}{lllllllll}4 & 7 & 1 & 8 & 5 & 0 & 6 & 3 & 2\end{array}$ $\begin{array}{lllllllll}5 & 8 & 2 & 6 & 3 & 1 & 7 & 4 & 0\end{array}$

* $6 \begin{array}{lllllllll}6 & 0 & 3 & 4 & 2 & 7 & 5 & 1 & 8\end{array}$ $\begin{array}{lllllllll}7 & 1 & 4 & 5 & 0 & 8 & 3 & 2 & 6\end{array}$ $\begin{array}{lllllllll}8 & 2 & 5 & 3 & 1 & 6 & 4 & 0 & 7\end{array}$

$\begin{array}{llllllllll}* & 0 & 5 & 7 & 8 & 3 & 2 & 4 & 1 & 6\end{array}$ $\begin{array}{lllllllll}1 & 3 & 8 & 6 & 4 & 0 & 5 & 2 & 7\end{array}$ $\begin{array}{lllllllll}2 & 4 & 6 & 7 & 5 & 1 & 3 & 0 & 8\end{array}$ $\begin{array}{lllllllll}3 & 8 & 1 & 4 & 0 & 6 & 2 & 7 & 5\end{array}$ $\begin{array}{lllllllll}4 & 6 & 2 & 5 & 1 & 7 & 0 & 8 & 3\end{array}$

$\begin{array}{llllllllll}* & 5 & 7 & 0 & 3 & 2 & 8 & 1 & 6 & 4\end{array}$ $\begin{array}{lllllllll}6 & 2 & 4 & 1 & 7 & 5 & 8 & 3 & 0\end{array}$

$\begin{array}{llllllllll}* & 7 & 0 & 5 & 2 & 8 & 3 & 6 & 4 & 1\end{array}$

$\begin{array}{lllllllll}8 & 1 & 3 & 0 & 6 & 4 & 7 & 5 & 2\end{array}$

$\begin{array}{llllllllll}* & 0 & 7 & 5 & 4 & 6 & 1 & 8 & 2 & 3\end{array}$

$\begin{array}{lllllllll}1 & 8 & 3 & 5 & 7 & 2 & 6 & 0 & 4\end{array}$

$\begin{array}{lllllllll}2 & 6 & 4 & 3 & 8 & 0 & 7 & 1 & 5\end{array}$

$\begin{array}{lllllllll}3 & 1 & 8 & 2 & 5 & 7 & 4 & 6 & 0\end{array}$ $\begin{array}{lllllllll}4 & 2 & 6 & 0 & 3 & 8 & 5 & 7 & 1\end{array}$

$\begin{array}{llllllllll}* & 5 & 0 & 7 & 1 & 4 & 6 & 3 & 8 & 2\end{array}$ $\begin{array}{lllllllll}6 & 4 & 2 & 8 & 0 & 3 & 1 & 5 & 7\end{array}$

$\begin{array}{llllllllll}* & 7 & 5 & 0 & 6 & 1 & 4 & 2 & 3 & 8\end{array}$

$\begin{array}{lllllllll}8 & 3 & 1 & 7 & 2 & 5 & 0 & 4 & 6\end{array}$

$\begin{array}{llllllllll}* & 0 & 4 & 8 & 5 & 2 & 6 & 7 & 3 & 1\end{array}$ $\begin{array}{lllllllll}1 & 5 & 6 & 3 & 0 & 7 & 8 & 4 & 2\end{array}$ $\begin{array}{lllllllll}2 & 3 & 7 & 4 & 1 & 8 & 6 & 5 & 0\end{array}$ $\begin{array}{lllllllll}3 & 7 & 2 & 1 & 8 & 4 & 5 & 0 & 6\end{array}$

$\begin{array}{llllllllll}* & 4 & 8 & 0 & 2 & 6 & 5 & 3 & 1 & 7\end{array}$ $\begin{array}{lllllllll}5 & 6 & 1 & 0 & 7 & 3 & 4 & 2 & 8\end{array}$ $\begin{array}{lllllllll}6 & 1 & 5 & 7 & 3 & 0 & 2 & 8 & 4\end{array}$ $\begin{array}{lllllllll}7 & 2 & 3 & 8 & 4 & 1 & 0 & 6 & 5\end{array}$ $\begin{array}{llllllllll}* & 8 & 0 & 4 & 6 & 5 & 2 & 1 & 7 & 3\end{array}$ $\begin{array}{llllllllll}* & 0 & 6 & 3 & 1 & 5 & 8 & 2 & 4 & 7\end{array}$ $\begin{array}{lllllllll}1 & 7 & 4 & 2 & 3 & 6 & 0 & 5 & 8\end{array}$ $\begin{array}{lllllllll}2 & 8 & 5 & 0 & 4 & 7 & 1 & 3 & 6\end{array}$ $\begin{array}{llllllllll}* & 3 & 0 & 6 & 8 & 1 & 5 & 7 & 2 & 4\end{array}$ $\begin{array}{lllllllll}4 & 1 & 7 & 6 & 2 & 3 & 8 & 0 & 5\end{array}$ $\begin{array}{lllllllll}5 & 2 & 8 & 7 & 0 & 4 & 6 & 1 & 3\end{array}$ $\begin{array}{llllllllll}* & 6 & 3 & 0 & 5 & 8 & 1 & 4 & 7 & 2\end{array}$ $\begin{array}{lllllllll}7 & 4 & 1 & 3 & 6 & 2 & 5 & 8 & 0\end{array}$ $\begin{array}{lllllllll}8 & 5 & 2 & 4 & 7 & 0 & 3 & 6 & 1\end{array}$

$\begin{array}{llllllllll}* & 0 & 8 & 4 & 7 & 1 & 3 & 5 & 6 & 2\end{array}$ $\begin{array}{lllllllll}1 & 6 & 5 & 8 & 2 & 4 & 3 & 7 & 0\end{array}$ $\begin{array}{lllllllll}2 & 7 & 3 & 6 & 0 & 5 & 4 & 8 & 1\end{array}$ $\begin{array}{lllllllll}3 & 2 & 7 & 5 & 6 & 0 & 1 & 4 & 8\end{array}$ $\begin{array}{llllllllll}* & 4 & 0 & 8 & 3 & 7 & 1 & 2 & 5 & 6\end{array}$ $\begin{array}{lllllllll}5 & 1 & 6 & 4 & 8 & 2 & 0 & 3 & 7\end{array}$ $\begin{array}{lllllllll}6 & 5 & 1 & 2 & 4 & 8 & 7 & 0 & 3\end{array}$ $\begin{array}{lllllllll}7 & 3 & 2 & 0 & 5 & 6 & 8 & 1 & 4\end{array}$ $\begin{array}{llllllllll}* & 8 & 4 & 0 & 1 & 3 & 7 & 6 & 2 & 5\end{array}$

Plane 1.44.1.2

$\begin{array}{llllllllll}* & 0 & 1 & 2 & 3 & 4 & 5 & 6 & 7 & 8\end{array}$

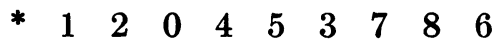

$\begin{array}{llllllllll}* & 2 & 0 & 1 & 5 & 3 & 4 & 8 & 6 & 7\end{array}$

$\begin{array}{llllllllll}* & 3 & 4 & 5 & 6 & 7 & 8 & 0 & 1 & 2\end{array}$

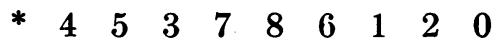

$\begin{array}{llllllllll}* & 5 & 3 & 4 & 8 & 6 & 8 & 2 & 0 & 1\end{array}$

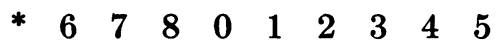

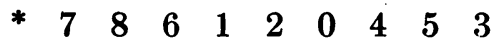

$\begin{array}{llllllllll}* & 8 & 6 & 7 & 2 & 0 & 1 & 5 & 3 & 4\end{array}$

* $\begin{array}{lllllllll} & 3 & 6 & 2 & 7 & 4 & 1 & 8 & 5\end{array}$ $\begin{array}{lllllllll}1 & 8 & 5 & 0 & 3 & 6 & 2 & 7 & 1\end{array}$ $\begin{array}{lllllllll}2 & 7 & 4 & 1 & 8 & 5 & 0 & 3 & 6\end{array}$

$\begin{array}{llllllllll}* & 3 & 6 & 0 & 7 & 4 & 2 & 8 & 5 & 1\end{array}$ $\begin{array}{lllllllll}4 & 2 & 7 & 5 & 1 & 8 & 6 & 0 & 3\end{array}$ $\begin{array}{llllllllll}* & 0 & 2 & 1 & 6 & 8 & 7 & 3 & 5 & 4\end{array}$

$\begin{array}{llllllllll}* & 1 & 0 & 2 & 7 & 6 & 8 & 4 & 3 & 5\end{array}$

$\begin{array}{llllllllll}* & 2 & 1 & 0 & 8 & 7 & 6 & 5 & 4 & 3\end{array}$

$\begin{array}{llllllllll}* & 3 & 5 & 4 & 0 & 2 & 1 & 6 & 8 & 7\end{array}$

$\begin{array}{llllllllll}* & 4 & 3 & 5 & 1 & 0 & 2 & 7 & 6 & 8\end{array}$

$\begin{array}{llllllllll}* & 5 & 4 & 3 & 2 & 1 & 0 & 8 & 7 & 6\end{array}$

* $6 \begin{array}{lllllllll} & 6 & 7 & 3 & 5 & 4 & 0 & 2 & 1\end{array}$

$\begin{array}{llllllllll}* & 7 & 6 & 8 & 4 & 3 & 5 & 1 & 0 & 2\end{array}$

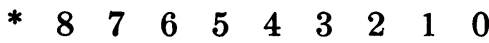

$\begin{array}{llllllllll}* & 0 & 4 & 8 & 5 & 2 & 6 & 7 & 3 & 1\end{array}$

$\begin{array}{lllllllll}1 & 7 & 3 & 8 & 0 & 4 & 6 & 5 & 2\end{array}$

$\begin{array}{lllllllll}2 & 6 & 5 & 3 & 1 & 7 & 4 & 8 & 0\end{array}$

$\begin{array}{lllllllll}3 & 1 & 7 & 4 & 8 & 0 & 2 & 6 & 5\end{array}$

$\begin{array}{llllllllll}* & 4 & 8 & 0 & 2 & 6 & 5 & 3 & 1 & 7\end{array}$

Note: Starred lines are common to 1.44.1.1 and 1.44.1.2. 
Plane 1.44.1.2-continued

\begin{tabular}{|c|c|c|c|c|c|c|c|c|c|c|c|c|c|c|c|c|c|c|}
\hline 5 & 1 & 8 & 6 & 0 & 3 & 4 & 2 & 7 & & 5 & 2 & 6 & 7 & 3 & 1 & 0 & 4 & 8 \\
\hline 6 & 0 & 3 & 4 & 2 & 7 & 5 & 1 & 8 & & 6 & 5 & 2 & 1 & 7 & 3 & 8 & 0 & 4 \\
\hline 7 & 4 & 2 & 8 & 5 & 1 & 3 & 6 & 0 & & 7 & 3 & 1 & 0 & 4 & 8 & 5 & 2 & 6 \\
\hline 8 & 5 & 1 & 3 & 6 & 0 & 7 & 4 & 2 & * & 8 & 0 & 4 & 6 & 5 & 2 & 1 & 7 & \\
\hline 0 & 5 & 7 & 8 & 3 & 2 & 4 & 1 & 6 & $*$ & 0 & 6 & 3 & 1 & 5 & 8 & 2 & 4 & \\
\hline 1 & 6 & 4 & 5 & 7 & 0 & 3 & 2 & 8 & & 1 & 5 & 8 & 2 & 4 & 7 & 0 & 6 & \\
\hline 2 & 8 & 3 & 6 & 4 & 1 & 7 & 0 & 5 & & 2 & 4 & 7 & 0 & 6 & 3 & 1 & 5 & \\
\hline 3 & 2 & 8 & 1 & 6 & 4 & 5 & 7 & 0 & $*$ & 3 & 0 & 6 & 8 & 1 & 5 & 7 & 2 & \\
\hline 4 & 1 & 6 & 0 & 5 & 7 & 8 & 3 & 2 & & 4 & 7 & 2 & 6 & 3 & 0 & 5 & 8 & 1 \\
\hline 5 & 7 & 0 & 3 & 2 & 8 & 1 & 6 & 4 & & 5 & 8 & 1 & 4 & 7 & 2 & 6 & 3 & \\
\hline 6 & 4 & 1 & 7 & 0 & 5 & 2 & 8 & 3 & $*$ & 6 & 3 & 0 & 5 & 8 & 1 & 4 & 7 & 2 \\
\hline 7 & 0 & 5 & 2 & 8 & 3 & 6 & 4 & 1 & & 7 & 2 & 4 & 3 & 0 & 6 & 8 & 1 & 5 \\
\hline 8 & 3 & 2 & 4 & 1 & 6 & 0 & 5 & 7 & & 8 & 1 & 5 & 7 & 2 & 4 & 3 & 0 & 6 \\
\hline 0 & 7 & 5 & 4 & 6 & 1 & 8 & 2 & 3 & $*$ & 0 & 8 & 4 & 7 & 1 & 3 & 5 & 6 & \\
\hline 1 & 4 & 6 & 3 & 8 & 2 & 5 & 0 & 7 & & 1 & 3 & 7 & 6 & 2 & 5 & 8 & 4 & C \\
\hline 2 & 3 & 8 & 7 & 5 & 0 & 6 & 1 & 4 & & 2 & 5 & 6 & 4 & 0 & 8 & 3 & 7 & 1 \\
\hline 3 & 8 & 2 & 5 & 0 & 7 & 1 & 4 & 6 & & 3 & 7 & 1 & 2 & 5 & 6 & 4 & 0 & 8 \\
\hline 4 & 6 & 1 & 8 & 2 & 3 & 0 & 7 & 5 & $*$ & 4 & 0 & 8 & 3 & 7 & 1 & 2 & 5 & \\
\hline 5 & 0 & 7 & 1 & 4 & 6 & 3 & 8 & 2 & & 5 & 6 & 2 & 0 & 8 & 4 & 7 & 1 & 3 \\
\hline 6 & 1 & 4 & 2 & 3 & 8 & 7 & 5 & 0 & & 6 & 2 & 5 & 8 & 4 & 0 & 1 & 3 & 7 \\
\hline 7 & 5 & 0 & 6 & 1 & 4 & 2 & 3 & 8 & & 7 & 1 & 3 & 5 & 6 & 2 & 0 & 8 & \\
\hline 8 & 2 & 3 & 0 & 7 & 5 & 4 & 6 & 1 & $*$ & 8 & 4 & 0 & 1 & 3 & 7 & 6 & 2 & \\
\hline
\end{tabular}

Plane 1.44.6

$\begin{array}{lllllllll}0 & 1 & 2 & 3 & 4 & 5 & 6 & 7 & 8\end{array}$

$\begin{array}{lllllllll}1 & 2 & 0 & 4 & 5 & 3 & 7 & 8 & 6\end{array}$

$\begin{array}{lllllllll}2 & 0 & 1 & 5 & 3 & 4 & 8 & 6 & 7\end{array}$

$\begin{array}{lllllllll}3 & 4 & 5 & 6 & 7 & 8 & 0 & 1 & 2\end{array}$

$\begin{array}{lllllllll}4 & 5 & 3 & 7 & 8 & 6 & 1 & 2 & 0\end{array}$

$\begin{array}{lllllllll}5 & 3 & 4 & 8 & 6 & 7 & 2 & 0 & 1\end{array}$

$\begin{array}{lllllllll}6 & 7 & 8 & 0 & 1 & 2 & 3 & 4 & 5\end{array}$

$\begin{array}{lllllllll}7 & 8 & 6 & 1 & 2 & 0 & 4 & 5 & 3\end{array}$

$\begin{array}{lllllllll}8 & 6 & 7 & 2 & 0 & 1 & 5 & 3 & 4\end{array}$

$\begin{array}{lllllllll}0 & 3 & 6 & 2 & 7 & 4 & 1 & 8 & 5\end{array}$

$\begin{array}{lllllllll}1 & 8 & 3 & 5 & 6 & 2 & 0 & 7 & 4\end{array}$

$\begin{array}{lllllllll}2 & 6 & 4 & 0 & 8 & 5 & 7 & 1 & 3\end{array}$

$\begin{array}{lllllllll}3 & 0 & 8 & 7 & 4 & 1 & 2 & 5 & 6\end{array}$

$\begin{array}{lllllllll}4 & 2 & 5 & 8 & 1 & 0 & 6 & 3 & 7\end{array}$

$\begin{array}{lllllllll}5 & 1 & 7 & 6 & 2 & 3 & 8 & 4 & 0\end{array}$

$\begin{array}{lllllllll}6 & 4 & 0 & 1 & 3 & 7 & 5 & 2 & 8\end{array}$

$\begin{array}{lllllllll}7 & 5 & 2 & 4 & 0 & 8 & 3 & 6 & 1\end{array}$

$\begin{array}{lllllllll}8 & 7 & 1 & 3 & 5 & 6 & 4 & 0 & 2\end{array}$ $\begin{array}{lllllllll}0 & 2 & 1 & 6 & 8 & 7 & 3 & 5 & 4\end{array}$

$\begin{array}{lllllllll}1 & 0 & 2 & 8 & 7 & 6 & 5 & 4 & 3\end{array}$

$\begin{array}{lllllllll}2 & 1 & 0 & 7 & 6 & 8 & 4 & 3 & 5\end{array}$

$\begin{array}{lllllllll}3 & 8 & 7 & 0 & 5 & 4 & 6 & 2 & 1\end{array}$

$\begin{array}{lllllllll}4 & 7 & 6 & 5 & 0 & 3 & 2 & 1 & 8\end{array}$

$\begin{array}{lllllllll}5 & 6 & 8 & 4 & 3 & 0 & 1 & 7 & 2\end{array}$

$\begin{array}{lllllllll}6 & 5 & 4 & 3 & 2 & 1 & 0 & 8 & 7\end{array}$

$\begin{array}{lllllllll}7 & 4 & 3 & 2 & 1 & 5 & 8 & 0 & 6\end{array}$

$\begin{array}{lllllllll}8 & 3 & 5 & 1 & 4 & 2 & 7 & 6 & 0\end{array}$

$\begin{array}{lllllllll}0 & 4 & 8 & 5 & 2 & 6 & 7 & 3 & 1\end{array}$

$\begin{array}{lllllllll}1 & 7 & 4 & 2 & 3 & 8 & 6 & 5 & 0\end{array}$

$\begin{array}{lllllllll}2 & 8 & 5 & 3 & 0 & 7 & 1 & 4 & 6\end{array}$

$\begin{array}{lllllllll}3 & 1 & 6 & 4 & 8 & 2 & 5 & 0 & 7\end{array}$

$\begin{array}{lllllllll}4 & 0 & 7 & 1 & 6 & 5 & 3 & 8 & 2\end{array}$

$\begin{array}{lllllllll}5 & 2 & 3 & 0 & 7 & 1 & 4 & 6 & 8\end{array}$

$\begin{array}{lllllllll}6 & 3 & 2 & 7 & 5 & 0 & 8 & 1 & 4\end{array}$

$\begin{array}{lllllllll}7 & 6 & 1 & 8 & 4 & 3 & 0 & 2 & 5\end{array}$

$\begin{array}{lllllllll}8 & 5 & 0 & 6 & 1 & 4 & 2 & 7 & 3\end{array}$

Note Starred lines are common to 1.44.1.1 and 1.44.1.2. 


\section{Plane 1.44.6-continued}

$\begin{array}{lllllllll}0 & 5 & 7 & 8 & 3 & 2 & 4 & 1 & 6 \\ 1 & 6 & 5 & 7 & 2 & 4 & 3 & 0 & 8 \\ 2 & 7 & 3 & 6 & 4 & 0 & 5 & 8 & 1 \\ 3 & 2 & 4 & 1 & 0 & 6 & 8 & 7 & 5 \\ 4 & 1 & 8 & 2 & 5 & 7 & 0 & 6 & 3 \\ 5 & 0 & 6 & 3 & 1 & 8 & 7 & 2 & 4 \\ 6 & 8 & 1 & 4 & 7 & 5 & 2 & 3 & 0 \\ 7 & 3 & 0 & 5 & 8 & 1 & 6 & 4 & 2 \\ 8 & 4 & 2 & 0 & 6 & 3 & 1 & 5 & 7 \\ 0 & 7 & 5 & 4 & 6 & 1 & 8 & 2 & 3 \\ 1 & 4 & 7 & 3 & 8 & 0 & 2 & 6 & 5 \\ 2 & 5 & 8 & 1 & 7 & 3 & 6 & 0 & 4 \\ 3 & 6 & 2 & 5 & 1 & 7 & 4 & 8 & 0 \\ 4 & 3 & 1 & 0 & 2 & 8 & 5 & 7 & 6 \\ 5 & 8 & 0 & 2 & 4 & 6 & 3 & 1 & 7 \\ 6 & 1 & 3 & 8 & 0 & 4 & 7 & 5 & 2 \\ 7 & 0 & 4 & 6 & 5 & 2 & 1 & 3 & 8 \\ 8 & 2 & 6 & 7 & 3 & 5 & 0 & 4 & 1\end{array}$

$\begin{array}{lllllllll}0 & 6 & 3 & 1 & 5 & 8 & 2 & 4 & 7\end{array}$

$\begin{array}{lllllllll}1 & 5 & 6 & 0 & 4 & 7 & 8 & 3 & 2\end{array}$

$\begin{array}{lllllllll}2 & 3 & 7 & 4 & 1 & 6 & 0 & 5 & 8\end{array}$

$\begin{array}{lllllllll}3 & 7 & 0 & 8 & 2 & 5 & 1 & 6 & 4\end{array}$

$\begin{array}{lllllllll}4 & 8 & 2 & 6 & 3 & 1 & 7 & 0 & 5\end{array}$

$\begin{array}{lllllllll}5 & 4 & 1 & 7 & 0 & 2 & 6 & 8 & 3\end{array}$

$\begin{array}{lllllllll}6 & 0 & 5 & 2 & 8 & 3 & 4 & 7 & 1\end{array}$

$\begin{array}{lllllllll}7 & 2 & 8 & 3 & 6 & 4 & 5 & 1 & 0\end{array}$

$\begin{array}{lllllllll}8 & 1 & 4 & 5 & 7 & 0 & 3 & 2 & 6\end{array}$

$\begin{array}{lllllllll}0 & 8 & 4 & 7 & 1 & 3 & 5 & 6 & 2\end{array}$

$\begin{array}{lllllllll}1 & 3 & 8 & 6 & 0 & 5 & 4 & 2 & 7\end{array}$

$\begin{array}{lllllllll}2 & 4 & 6 & 8 & 5 & 1 & 3 & 7 & 0\end{array}$

$\begin{array}{lllllllll}3 & 5 & 1 & 2 & 6 & 0 & 7 & 4 & 8\end{array}$

$\begin{array}{lllllllll}4 & 6 & 0 & 3 & 7 & 2 & 8 & 5 & 1\end{array}$

$\begin{array}{lllllllll}5 & 7 & 2 & 1 & 8 & 4 & 0 & 3 & 6\end{array}$

$\begin{array}{lllllllll}6 & 2 & 7 & 5 & 4 & 8 & 1 & 0 & 3\end{array}$

$\begin{array}{lllllllll}7 & 1 & 5 & 0 & 3 & 6 & 2 & 8 & 4\end{array}$

$\begin{array}{lllllllll}8 & 0 & 3 & 4 & 2 & 7 & 6 & 1 & 5\end{array}$

Plane 1.4

$\begin{array}{lllllllll}0 & 1 & 2 & 3 & 4 & 5 & 6 & 7 & 8\end{array}$

$\begin{array}{lllllllll}1 & 2 & 0 & 4 & 5 & 3 & 7 & 8 & 6\end{array}$

$\begin{array}{lllllllll}2 & 0 & 1 & 5 & 3 & 4 & 8 & 6 & 7\end{array}$

$\begin{array}{lllllllll}3 & 4 & 5 & 6 & 7 & 8 & 0 & 1 & 2\end{array}$

$\begin{array}{lllllllll}4 & 5 & 3 & 7 & 8 & 6 & 1 & 2 & 0\end{array}$

$\begin{array}{lllllllll}5 & 3 & 4 & 8 & 6 & 7 & 2 & 0 & 1\end{array}$

$\begin{array}{lllllllll}6 & 7 & 8 & 0 & 1 & 2 & 3 & 4 & 5\end{array}$

$\begin{array}{lllllllll}7 & 8 & 6 & 1 & 2 & 0 & 4 & 5 & 3\end{array}$

$\begin{array}{lllllllll}8 & 6 & 7 & 2 & 0 & 1 & 5 & 3 & 4\end{array}$

$\begin{array}{lllllllll}0 & 3 & 5 & 2 & 1 & 6 & 4 & 8 & 7\end{array}$

$\begin{array}{lllllllll}1 & 6 & 4 & 5 & 2 & 8 & 3 & 7 & 0\end{array}$

$\begin{array}{lllllllll}2 & 8 & 3 & 0 & 4 & 7 & 5 & 1 & 6\end{array}$

$\begin{array}{lllllllll}3 & 0 & 8 & 1 & 6 & 5 & 7 & 2 & 4\end{array}$

$\begin{array}{lllllllll}4 & 7 & 1 & 8 & 5 & 0 & 6 & 3 & 2\end{array}$

$\begin{array}{lllllllll}5 & 1 & 7 & 4 & 8 & 2 & 0 & 6 & 3\end{array}$

$\begin{array}{lllllllll}6 & 4 & 2 & 7 & 0 & 3 & 8 & 5 & 1\end{array}$

$\begin{array}{lllllllll}7 & 5 & 0 & 6 & 3 & 1 & 2 & 4 & 8\end{array}$

$\begin{array}{lllllllll}8 & 2 & 6 & 3 & 7 & 4 & 1 & 0 & 5\end{array}$

$\begin{array}{lllllllll}0 & 5 & 4 & 1 & 7 & 2 & 8 & 3 & 6\end{array}$

$\begin{array}{lllllllll}1 & 7 & 3 & 2 & 6 & 4 & 0 & 5 & 8\end{array}$

$\begin{array}{lllllllll}2 & 6 & 5 & 3 & 8 & 0 & 7 & 4 & 1\end{array}$

$\begin{array}{lllllllll}3 & 8 & 1 & 4 & 0 & 6 & 2 & 7 & 5\end{array}$

$\begin{array}{lllllllll}4 & 1 & 8 & 6 & 2 & 3 & 5 & 0 & 7\end{array}$ $\begin{array}{lllllllll}0 & 2 & 1 & 6 & 8 & 7 & 3 & 5 & 4\end{array}$

$\begin{array}{lllllllll}1 & 0 & 2 & 8 & 7 & 6 & 5 & 4 & 3\end{array}$

$\begin{array}{lllllllll}2 & 1 & 0 & 7 & 6 & 8 & 4 & 3 & 5\end{array}$

$\begin{array}{lllllllll}3 & 5 & 7 & 0 & 2 & 4 & 6 & 8 & 1\end{array}$

$\begin{array}{lllllllll}4 & 3 & 6 & 5 & 0 & 2 & 7 & 1 & 8\end{array}$

$\begin{array}{lllllllll}5 & 4 & 8 & 2 & 3 & 0 & 1 & 7 & 6\end{array}$

$\begin{array}{lllllllll}6 & 8 & 4 & 3 & 5 & 1 & 0 & 2 & 7\end{array}$

$\begin{array}{lllllllll}7 & 6 & 3 & 4 & 1 & 5 & 8 & 0 & 2\end{array}$

$\begin{array}{lllllllll}8 & 7 & 5 & 1 & 4 & 3 & 2 & 6 & 0\end{array}$

$\begin{array}{lllllllll}0 & 4 & 3 & 8 & 2 & 1 & 7 & 6 & 5\end{array}$

$\begin{array}{lllllllll}1 & 8 & 5 & 7 & 3 & 2 & 6 & 0 & 4\end{array}$

$\begin{array}{lllllllll}2 & 7 & 4 & 6 & 0 & 5 & 1 & 8 & 3\end{array}$

$\begin{array}{lllllllll}3 & 1 & 6 & 2 & 5 & 7 & 8 & 4 & 0\end{array}$

$\begin{array}{lllllllll}4 & 0 & 7 & 3 & 1 & 8 & 2 & 5 & 6\end{array}$

$\begin{array}{lllllllll}5 & 6 & 1 & 0 & 7 & 3 & 4 & 2 & 8\end{array}$

$\begin{array}{lllllllll}6 & 3 & 0 & 1 & 8 & 4 & 5 & 7 & 2\end{array}$

$\begin{array}{lllllllll}7 & 2 & 8 & 5 & 4 & 6 & 0 & 3 & 1\end{array}$

$\begin{array}{lllllllll}8 & 5 & 2 & 4 & 6 & 0 & 3 & 1 & 7\end{array}$

$\begin{array}{lllllllll}0 & 6 & 8 & 7 & 5 & 4 & 2 & 1 & 3\end{array}$

$\begin{array}{lllllllll}1 & 3 & 7 & 6 & 4 & 0 & 8 & 2 & 5\end{array}$

$\begin{array}{lllllllll}2 & 5 & 6 & 8 & 1 & 3 & 0 & 7 & 4\end{array}$

$\begin{array}{lllllllll}3 & 7 & 2 & 5 & 8 & 1 & 4 & 0 & 6\end{array}$

$\begin{array}{lllllllll}4 & 8 & 0 & 2 & 7 & 5 & 3 & 6 & 1\end{array}$ 
Plane 1.4-continued

\begin{tabular}{|c|c|c|c|c|c|c|c|c|c|c|c|c|c|c|c|}
\hline 5 & 0 & 6 & 7 & 1 & 3 & 8 & 2 & 5 & 2 & 3 & 1 & 0 & 8 & 6 & 4 \\
\hline 6 & 2 & 7 & 8 & 5 & 4 & 1 & 0 & 6 & 0 & 5 & 4 & 2 & 7 & 1 & 3 \\
\hline 7 & 3 & 2 & 0 & 8 & 1 & 6 & 4 & 7 & 4 & 1 & 3 & 6 & 2 & 5 & 8 \\
\hline 8 & 4 & 0 & 5 & 7 & 6 & 2 & 3 & 8 & 1 & 4 & 0 & 3 & 6 & 7 & 5 \\
\hline 0 & 7 & 6 & 4 & 8 & 5 & 2 & 1 & 0 & 8 & 7 & 5 & 6 & 3 & 1 & 4 \\
\hline 1 & 5 & 8 & 3 & 7 & 4 & 6 & 2 & 1 & 4 & 6 & 0 & 8 & 5 & 2 & 3 \\
\hline 2 & 4 & 7 & 1 & 6 & 3 & 0 & 8 & 2 & 3 & 8 & 4 & 7 & 1 & 6 & 5 \\
\hline 3 & 6 & 0 & 8 & 2 & 1 & 5 & 7 & 3 & 2 & 4 & 7 & 1 & 0 & 5 & 6 \\
\hline 4 & 2 & 5 & 0 & 1 & 8 & 7 & 3 & 4 & 6 & 2 & 1 & 3 & 7 & 0 & 8 \\
\hline 5 & 8 & 2 & 6 & 4 & 7 & 3 & 0 & 5 & 7 & 0 & 3 & 2 & 6 & 8 & 1 \\
\hline 6 & 1 & 3 & 5 & 0 & 2 & 8 & 4 & 6 & 5 & 1 & 2 & 4 & 8 & 7 & 0 \\
\hline 7 & 0 & 4 & 2 & 3 & 6 & 1 & 5 & 7 & 1 & 5 & 8 & 0 & 4 & 3 & 2 \\
\hline & 3 & 1 & 7 & 5 & 0 & 4 & 6 & 8 & 0 & 3 & 6 & 5 & 2 & 4 & 7 \\
\hline
\end{tabular}

Plane 4.3

$\begin{array}{lllllllll}0 & 1 & 2 & 3 & 4 & 5 & 6 & 7 & 8\end{array}$

$\begin{array}{lllllllll}1 & 2 & 0 & 4 & 5 & 3 & 7 & 8 & 6\end{array}$

$\begin{array}{lllllllll}2 & 0 & 1 & 5 & 3 & 4 & 8 & 6 & 7\end{array}$

$\begin{array}{lllllllll}3 & 4 & 5 & 6 & 7 & 8 & 0 & 1 & 2\end{array}$

$\begin{array}{lllllllll}4 & 5 & 3 & 7 & 8 & 6 & 1 & 2 & 0\end{array}$

$\begin{array}{lllllllll}5 & 3 & 4 & 8 & 6 & 7 & 2 & 0 & 1\end{array}$

$\begin{array}{lllllllll}6 & 7 & 8 & 0 & 1 & 2 & 3 & 4 & 5\end{array}$

$\begin{array}{lllllllll}7 & 8 & 6 & 1 & 2 & 0 & 4 & 5 & 3\end{array}$

$\begin{array}{lllllllll}8 & 6 & 7 & 2 & 0 & 1 & 5 & 3 & 4\end{array}$

$\begin{array}{lllllllll}0 & 3 & 6 & 2 & 7 & 4 & 1 & 8 & 5\end{array}$

$\begin{array}{lllllllll}1 & 6 & 4 & 5 & 8 & 2 & 0 & 7 & 3\end{array}$

$\begin{array}{lllllllll}2 & 8 & 3 & 0 & 6 & 5 & 7 & 1 & 4\end{array}$

$\begin{array}{lllllllll}3 & 0 & 8 & 1 & 4 & 7 & 5 & 2 & 6\end{array}$

$\begin{array}{lllllllll}4 & 7 & 1 & 8 & 5 & 0 & 6 & 3 & 2\end{array}$

$\begin{array}{lllllllll}5 & 1 & 7 & 4 & 2 & 8 & 3 & 6 & 0\end{array}$

$\begin{array}{lllllllll}6 & 4 & 0 & 7 & 3 & 1 & 2 & 5 & 8\end{array}$

$\begin{array}{lllllllll}7 & 5 & 2 & 6 & 0 & 3 & 8 & 4 & 1\end{array}$

$\begin{array}{lllllllll}8 & 2 & 5 & 3 & 1 & 6 & 4 & 0 & 7\end{array}$

$\begin{array}{lllllllll}0 & 5 & 7 & 8 & 3 & 2 & 4 & 1 & 6\end{array}$

$\begin{array}{lllllllll}1 & 7 & 3 & 6 & 2 & 4 & 5 & 0 & 8\end{array}$

$\begin{array}{lllllllll}2 & 6 & 5 & 7 & 4 & 0 & 3 & 8 & 1\end{array}$

$\begin{array}{lllllllll}3 & 8 & 1 & 4 & 0 & 6 & 2 & 7 & 5\end{array}$

$\begin{array}{lllllllll}4 & 1 & 8 & 2 & 6 & 3 & 0 & 5 & 7\end{array}$

$\begin{array}{lllllllll}5 & 0 & 6 & 3 & 8 & 1 & 7 & 4 & 2\end{array}$

$\begin{array}{lllllllll}6 & 2 & 4 & 1 & 7 & 5 & 8 & 3 & 0\end{array}$

$\begin{array}{lllllllll}7 & 3 & 0 & 5 & 1 & 8 & 6 & 2 & 4\end{array}$

$\begin{array}{lllllllll}8 & 4 & 2 & 0 & 5 & 7 & 1 & 6 & 3\end{array}$ $\begin{array}{lllllllll}0 & 2 & 1 & 7 & 6 & 8 & 5 & 4 & 3\end{array}$

$\begin{array}{lllllllll}1 & 0 & 2 & 8 & 7 & 6 & 3 & 5 & 4\end{array}$

$\begin{array}{lllllllll}2 & 1 & 0 & 6 & 8 & 7 & 4 & 3 & 5\end{array}$

$\begin{array}{lllllllll}3 & 5 & 4 & 2 & 1 & 0 & 7 & 6 & 8\end{array}$

$\begin{array}{lllllllll}4 & 3 & 5 & 0 & 2 & 1 & 8 & 7 & 6\end{array}$

$\begin{array}{lllllllll}5 & 4 & 3 & 1 & 0 & 2 & 6 & 8 & 7\end{array}$

$\begin{array}{lllllllll}6 & 8 & 7 & 5 & 4 & 3 & 1 & 0 & 2\end{array}$

$\begin{array}{lllllllll}7 & 6 & 8 & 3 & 5 & 4 & 2 & 1 & 0\end{array}$

$\begin{array}{lllllllll}8 & 7 & 6 & 4 & 3 & 5 & 0 & 2 & 1\end{array}$

$\begin{array}{lllllllll}0 & 4 & 8 & 5 & 2 & 6 & 7 & 3 & 1\end{array}$

$\begin{array}{lllllllll}1 & 8 & 5 & 2 & 3 & 7 & 6 & 4 & 0\end{array}$

$\begin{array}{lllllllll}2 & 7 & 4 & 3 & 0 & 8 & 1 & 5 & 6\end{array}$

$\begin{array}{lllllllll}3 & 1 & 6 & 7 & 5 & 2 & 8 & 0 & 4\end{array}$

$\begin{array}{lllllllll}4 & 0 & 7 & 6 & 1 & 5 & 2 & 8 & 3\end{array}$

$\begin{array}{lllllllll}5 & 6 & 1 & 0 & 7 & 3 & 4 & 2 & 8\end{array}$

$\begin{array}{lllllllll}6 & 3 & 2 & 4 & 8 & 0 & 5 & 1 & 7\end{array}$

$\begin{array}{lllllllll}7 & 2 & 3 & 8 & 4 & 1 & 0 & 6 & 5\end{array}$

$\begin{array}{lllllllll}8 & 5 & 0 & 1 & 6 & 4 & 3 & 7 & 2\end{array}$

$\begin{array}{lllllllll}0 & 6 & 3 & 4 & 1 & 7 & 8 & 5 & 2\end{array}$

$\begin{array}{lllllllll}1 & 5 & 6 & 0 & 4 & 8 & 2 & 3 & 7\end{array}$

$\begin{array}{lllllllll}2 & 3 & 7 & 1 & 5 & 6 & 0 & 4 & 8\end{array}$

$\begin{array}{lllllllll}3 & 7 & 2 & 5 & 6 & 1 & 4 & 8 & 0\end{array}$

$\begin{array}{lllllllll}4 & 8 & 0 & 3 & 7 & 2 & 5 & 6 & 1\end{array}$

$\begin{array}{lllllllll}5 & 2 & 8 & 6 & 3 & 0 & 1 & 7 & 4\end{array}$

$\begin{array}{lllllllll}6 & 1 & 5 & 8 & 0 & 4 & 7 & 2 & 3\end{array}$

$\begin{array}{lllllllll}7 & 4 & 1 & 2 & 8 & 5 & 3 & 0 & 6\end{array}$

$\begin{array}{lllllllll}8 & 0^{\circ} & 4 & 7 & 2 & 3 & 6 & 1 & 5\end{array}$ 


\section{Plane 4.3-continued}

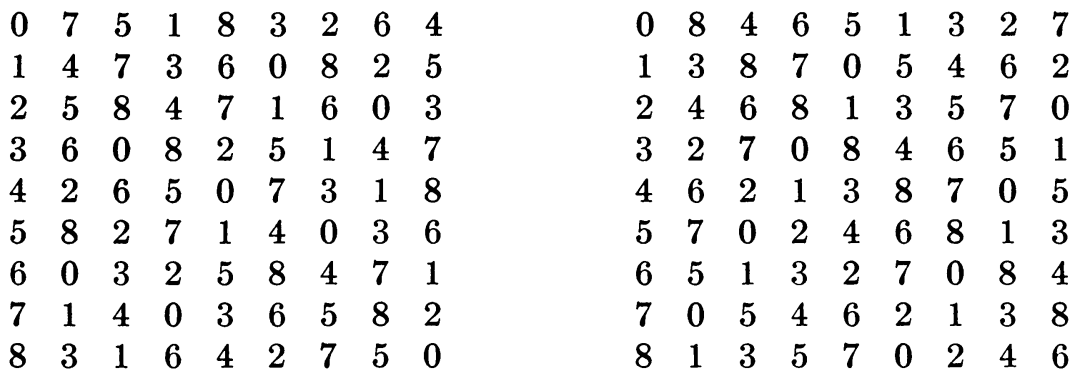

7. Concluding remarks. The failure to find, in this extensive search, any planes beyond those known for many years strengthens the conjecture that the four planes are a complete set for order 9 . This number is the smallest order still in doubt as to the list of possible planes and also is the smallest order with more than a single plane [2]. It is still not possible within the limits of present mechanical equipment to make a complete search for order 9 as was done for 8 . For one thing, no list of latin squares of order 8 yet exists. It is planned to continue the search with other likely additive pencils beginning with the cyclic group.

The reader will have noted that, whereas the procedures used in the basic search were mechanized or easily mechanizable, the methods of section 6 were more involved and clearly ad hoc. No satisfactory mechanical way to identify two isomorphic planes exists whether they be presented by a coordinate system or by an incidence matrix. The preparation of such a method is an interesting question.

University of California, Los Angeles, California, Ohio State University, Columbus, Ohio

1. Marshall Hall, JR., "Projective planes," Trans. Amer. Math. Soc., v. 54, 1943, p. 229-277.

2. Marshall Hall, Jr., J. Dean Swift \& Robert J. Walker, "Uniqueness of the projective plane of order eight," MTAC, v. 10, 1956, p. 186-194.

3. Daniel Hughes, "A class of non-desarguesian projective planes," Canad. J. Math., v. 9,1957, p. $378-388$.

4. O. VEBlen \& J. H. MacLagan-Wedderburn, "Non-desarguesian and non-pascalian geometries," Trans. Amer. Math. Soc., v. 8, 1907, p. 379-388. 